\title{
CASE REPORT: BILATERAL DAMAGE OF THE INFERIOR ALVEOLAR NERVES AFTER DENTAL IMPLANT PLACEMENT
}

\author{
N. Firfiris1, E. Moka2, G. Micha1, S. Poulopoulou1, K. Kappatos3, E. Vrachnou1. \\ 1."Agios Savvas" Anticancer Hospital, Department of Anaesthesiology and Pain Therapy, \\ Athens, Greece \\ 2.Creta InterClinic Hospital, Anaesthesiology Department, Heraklion- Crete, Greece. \\ 3.Dentists' Institute, Dentist's Private Office, Athens, Greece
}

\section{Background and Aims}

Several complications during dental implant placement have been recognized, including nerve injury. Inferior alveolar nerve damage seems to prevail, yet there are no conclusive data regarding incidence and management of subsequent provoked neuropathic orofacial pain.

\section{Methods}

Case report: A 70 year old female patient presented in the Pain Center of our Hospital with a one-month history of burning sensation in the inferior lip, following 4 dental implants placement. During the procedure she described an excruciating pain, numbness and a burning sensation, being at that time managed with NSAIDs, that proved to be inefficient. 48 hours later a CT scan revealed a severe bilateral injury of the inferior alveolar nerves and a left inferior alveolar artery puncture (Figure 1,2). As such implants were extracted 15 days later.

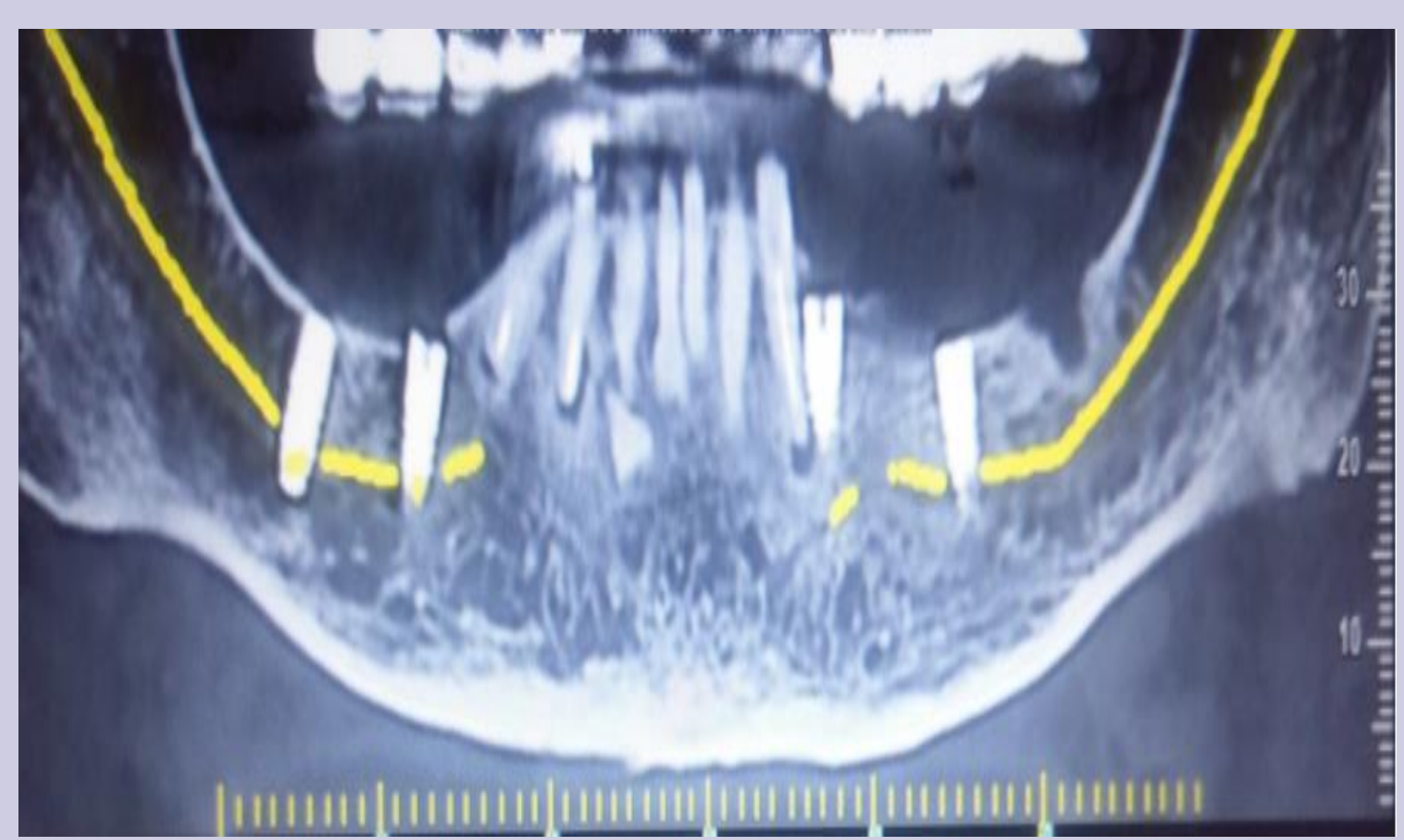

Figure 1. CT scan of inferior mandible

\section{Results}

At the time of clinical assessment in the Pain Center, patient presented with numbness and a burning sensation in the inferior lip with absence of cutaneous sensation, as assessed by pinprick and temperature sensitivity tests. Pain intensity score was 9 (NPRS) and the patient was prescribed treatment with pregabalin and duloxetine for pain management. In the first follow-up she reported a decreased pain intensity (NPRS 7) yet the numbness and the burning sensation were still present. It was then decided to bilaterally infiltrate the inferior alveolar nerves with dexamethasone (4mg).

\section{Conclusions}

Fifteen days later all symptoms abated (NPRS 3) and two months later carbamazepine was added to the analgesic scheme. Seven months after initial assessment patient was symptoms free and a stepping down pain treatment followed.

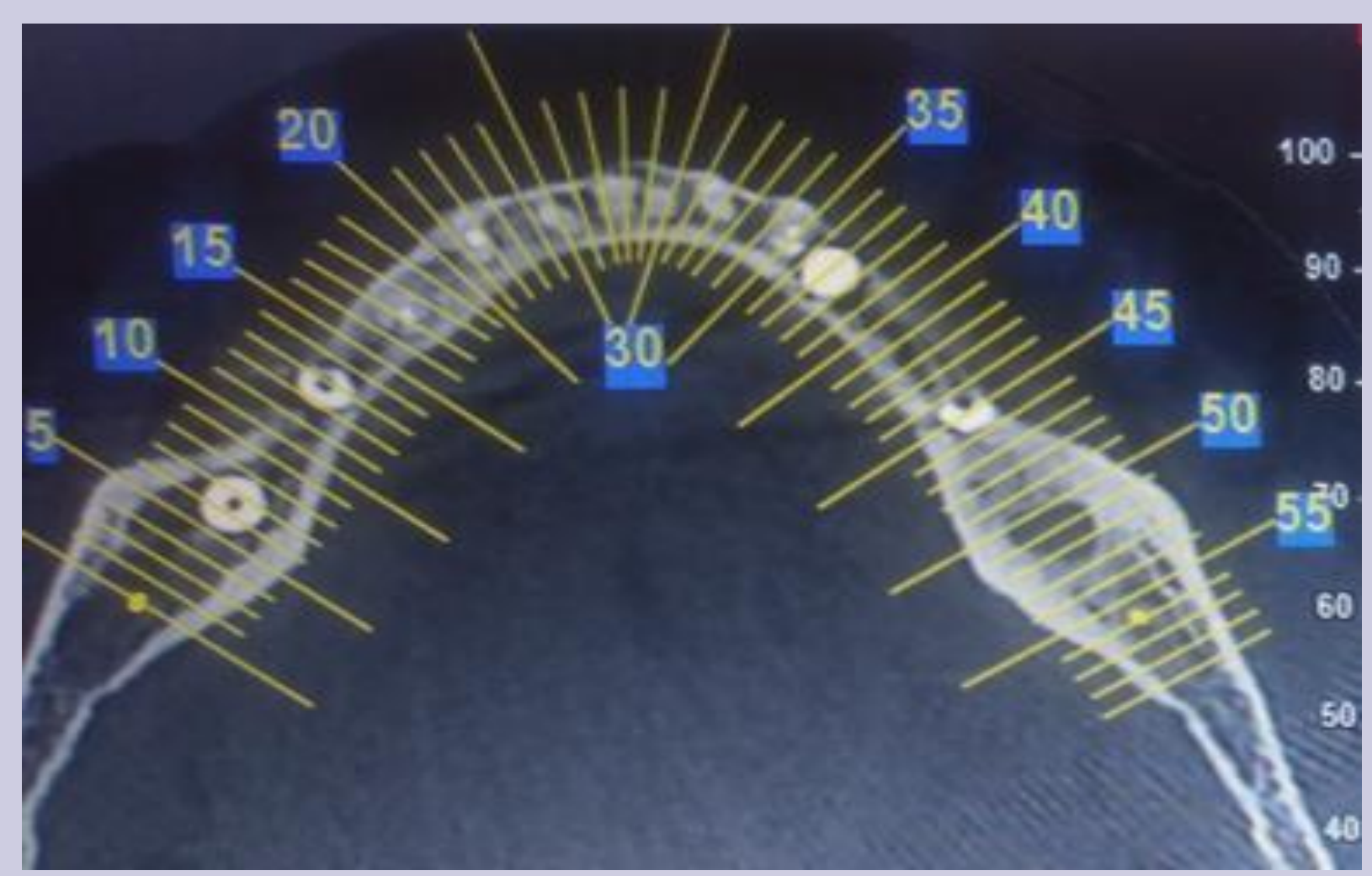

Figure 2. CT scan of inferior mandible-transverse view

Reference:

Kim YT et al. Clinical outcome of conservative treatment of injured inferior alveolar nerve during dental implant placement. $\mathbf{J}$ Korean Assoc Oral Maxillofac Surg. 2013 Jun; 39(3): 127-133 